\title{
Research on Optimizing Strategy of Distribution Network Operation Efficiency Based on PSO
}

\author{
Tao Han ${ }^{1, \text { a }}$, Shaoguang Yuan ${ }^{2, b}$, Quan Zhou ${ }^{3, c}$, Kun Yu ${ }^{3, d}$ \\ ${ }^{1}$ NARI Group/State Grid Electric Power Research Institute Corporation, Nanjing 211000, Jiangsu, \\ China \\ ${ }^{2}$ State Grid Henan Electric Power Company Electric Power Research Institute, Zhengzhou 450052, \\ Henan, China \\ ${ }^{3}$ College of Energy and Electrical Engineering, Hohai University, Nanjing 211100, China \\ ataohan@163.com, bsgyuan@163.com, 'zq0901zy@163.com, dkun.yu@vip.sina.com
}

Keywords: Distribution network; Dynamic reconfiguration; Distributed generation.

Abstract. Closed operation control of section switch and loop switch in distribution network to change the topology structure of the distribution network, thereby changing the distribution of power flows in the distribution network. It can improve the operating efficiency of the distribution network. In addition, with the continuous increase in the penetration rate of distributed power in distribution networks, the adjustment of distributed power output can change the flow on the line and affect the operational efficiency of the distribution network. This paper proposes the optimization method of distribution network operating efficiency from the perspective of operation optimization, mainly including network reconstruction and distributed power supply output regulation. The distribution characteristics of distributed power supply in distribution network are analyzed. The output and the state of the distribution network switch are used as control variables, and the network loss and load balance of the system are used as objective functions to study the comprehensive improvement measures for the distribution network operating efficiency.

\section{Introduction}

From the perspective of distribution network, the power elements include upper power supply ${ }^{[1]}$, distributed generation ${ }^{[2]}(\mathrm{DG})$, micro grid ${ }^{[3]}(\mathrm{MG})$ etc. Changes in the output of the power supply element will affect the optimal operation plan of the distribution network. Changes in the operation status of the distribution network will affect the load distribution of the up-per-level power supply and the regulation of the voltage quality of the distributed power supply. That is, the source and network is affect each other.

The research of existing literature focuses on net-work reconstruction problems with DG, and most of them have the objective function of minimizing network loss or minimizing voltage offset. The literature [4] pro-posed an integrated optimization model for network reconfiguration and DG output based on PSO algorithm. The weighted method was used to convert the two goals with the lowest network loss and the smallest voltage offset into single-target problems. The literature [5] uses cross-iteration to optimize the DG output and the net-work operating status. Actual examples show that this method can achieve the overall optimality of the distribution network. The literature [6], the ant colony algorithm is used to achieve the simultaneous optimization of network reconfiguration and schedulable DG, but it is necessary to satisfy the radial constraints of the distribution network.

The above literature studies the distribution net-work scheduling technology that takes into account DG. Both the DG output and the switching behavior of the distribution network are used as the optimization variables, or both are optimized or iteratively optimized to obtain the optimal solution. However, the impact of the elements such as the upper power supply and microgrid on distribution network scheduling is less considered, and the effect of the voltage regulation of the DG on the improvement of the distribution network voltage is not taken into account, and the maintenance and power maintenance existing in the actual scheduling process of the distribution network are not taken into consideration. 
This paper analyzes the output characteristics of distributed power supply in distribution network, classifies the distributed power supply according to its controllability, and studies the influence of reactive power compensation and network reconfiguration in distribution network on the distribution network operating efficiency. The active power output and the state of the distribution network switch are used as the control variables, and the network loss and load balance of the system are taken as objective functions to study the comprehensive improvement measures of the distribution network operating efficiency.

\section{Distribution Network Operation Efficiency Optimization Model}

Objective function. (1) Network loss

$$
\min \operatorname{Loss}=\sum_{t=1}^{24} \sum_{l=1}^{N_{b}} u_{l} \cdot\left(I_{l}^{t}\right)^{2} \cdot R_{l}
$$

Where, $l=1,2, \mathrm{~L} N_{b}, N_{b}$ is the total number of branches, $u_{l}$ is the state of the branch $l$, the branch power supply is 1 , otherwise it is $0, R_{l}$ represents the resistance of the branch $l$.

(2)Load balancing

Load balance is used to measure the fluctuation of the load of each substation in the whole distribution network. The calculation formula for this indicator is as follows:

$$
\min V O=\sum_{x=1}^{N_{s}}\left(\sum_{t=1}^{23}\left|P_{\text {total }, x}^{(t+1)}-P_{\text {total }, x}^{t}\right| / \sum_{t=1}^{24} P_{\text {total }, x}^{t}\right)
$$

Where: $V O$ is the integrated load fluctuation coefficient of the distribution network, $N$ is the number of upper substations in the distribution network of the studied area; $P_{t o t a l, x}^{t}$ is the load value of the $x$ substation at time $t, \mathrm{~kW}$, the value is related to the topology of the network, the load of the grid, the controllable DG, the output of the photovoltaic power supply, and the network loss.

(3)The Number of Switching Operations

$$
\min N S_{\text {total }}=\sum_{n=1}^{N} \sum_{i=1}^{S W}\left|k_{i}^{n+1}-k_{i}^{n}\right|
$$

Where, $S W$ is the total number of segmented switches and associated switches in the distribution network, $N$ is the number of reconstructions, $k_{i}^{n+1} 、 k_{i}^{n}$ represents the state of the switches before and after reconstruction.

Restrictions. Power Flow Constraints, Branch Current Constraints, Node voltage constraints, the capacity constraints of the upper power supply, the local consumption constraints of the photovoltaic power supply, the voltage adjustment constraints/capacity constraints of the controllable DG, the switching power constraints of the micro grid.

\section{Solution Process Based on Multi-objective Particle Swarm Optimization}

MOPSO uses external particle swarms to store Pareto non-dominated solutions in the search and constantly updates them. The adaptive grid method was used to evaluate the density information of nondominated solutions in the outer particle swarm. The mini-mum density Pareto nondominated solution was chosen as the Gbest guided particle optimization. It diversified the Pareto nondominated solutions and made the optimal frontier distribution uniform. 
The basic idea of using a particle algorithm to solve the comprehensive optimization strategy of distribution network operating efficiency is to use the power moment method to divide the reconstruction time of the distribution network, and then take the DG active output and the switch state as control variables and substitute the particles Group algorithm. The specific algorithm flow is as follows:

(1) According to the data of the load in the distribution network, calculate the unbalanced power moment of the distribution network, and then divide the reconstruction period.

(2) Simplified analysis and processing of the distribution network.

(3) The position and velocity of the particle swarm are initialized. There are three variables optimized in this paper. Select one of the five basic loops in turn and turn off the switch and set the other switches to be inoperable. In reactive power optimization, the number of initializing capacitor inputs is set; when DG active injection is optimized, the active power of DG is initialized within the DG output limit.

(4) The active power of the population formed by the switch state in the distribution network and the DG are taken as the independent variables in the network reconstruction, and it is checked whether the network satisfies the radial constraint, and then the power flow is calculated, and the expected value of the calculated objective function is taken as the fitness value in particle swarm.

(5) The update history is selected according to the individual extremum of the current position; if the individual extremum with particles is superior to the global extremum of the population, then the individual extremum is used to replace the original global extremum of the particle.

(6) Update the speed and position of particles in a particle swarm.

(7) Determine whether the particle swarm algorithm reaches the set maximum number of iterations. If yes, stop iterating, otherwise, return to step (4).

\section{Analysis of Examples}

In order to verify the improvement of the distribution network operation efficiency proposed in this paper, this paper validates the algorithm proposed in this paper according to the complex distribution network of the 94-node multi-station substation. The distribution network has a voltage rating of 10 $\mathrm{kV}$, a total of 11 feeders, 83 segment switches and 13 contact switches. The wiring diagram of the distribution network is shown in Fig. 1.

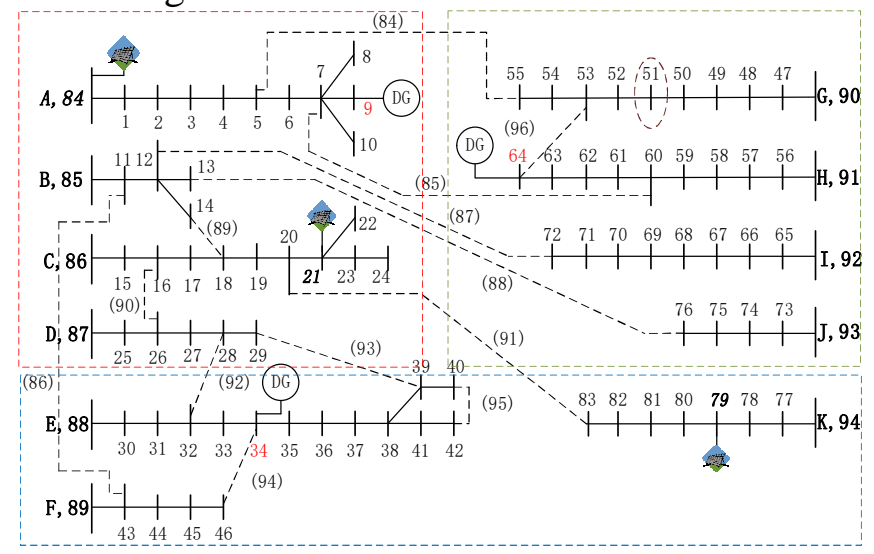

Fig. 1 Complex Distribution Network Structure

Substation 1 has two main transformers, each with a capacity of 12.5 MVA. Substation 2 and Substation 3 are also two main transformers, each with 8 MVA. The spare coefficient k takes 0.75 . In the substation 1 power supply area, photovoltaic power is installed at A and node 21 , and a controllable DG is installed at node 9. In the substation 2 power supply area, photovoltaic power is installed at node 79, and a controllable DG is installed at node 42. In the power supply area of the substation 3, a controllable DG is installed at the node 72. Randomly allocate the load of residential, commercial, and industrial users to the standard 94-node study. 
The DG capacity at location A is $1000 \mathrm{~kW}$, and the PV power supply capacity at node 21 and node 79 is $200 \mathrm{~kW}$. The output curve of photovoltaic power supply is shown in Fig. 2. Photovoltaic power generation is an uncontrollable DG. The principle of local consumption is adopted for such clean energy.

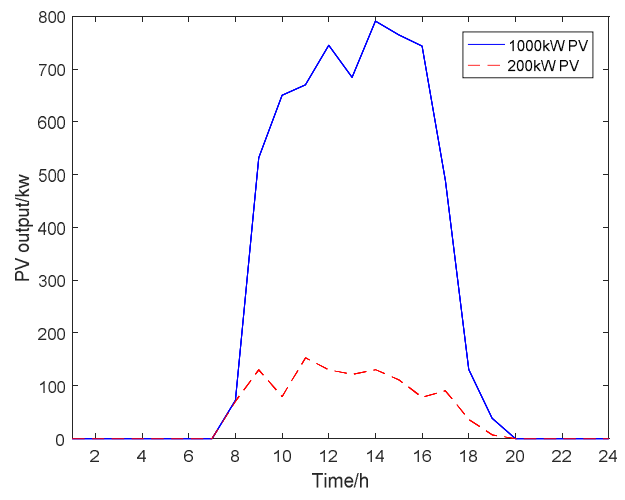

Fig. 2 PV output forecast

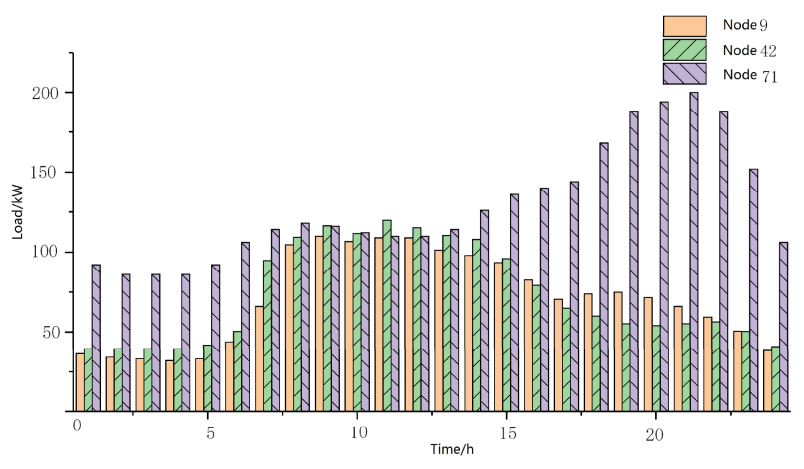

Fig. 3 Distributed Power Output Regulation

Let the number of particles in the PSO be 200, the maximum number of iterations be 300 , the learning factor set to 2 , and the inertia weight coefficients 1.1, 0.8. Encode the particles. The resulting distributed power output is shown in Fig. 3.

Table 1 Network Reconstruction Scheme

\begin{tabular}{cccc}
\hline $\begin{array}{c}\text { Optimized } \\
\text { scheme }\end{array}$ & Optimized period & Network Reconstruction Scheme & Optimized period \\
\hline \multirow{2}{*}{ Scheme 1} & $8: 00-21: 00$ & {$[52,85,86,72,88,89,90,83,27,29,33,40,55]$} & $8: 00-15: 00$ \\
& $22: 00-7: 00$ & {$[52,85,86,70,88,89,90,83,27,29,33,40,55]$} & $22: 00-7: 00$ \\
& $8: 00-21: 00$ & {$[84,85,86,87,88,89,90,91,27,93,94,95,52]$} & $8: 00-15: 00$ \\
Scheme 2 & $22: 00-7: 00$ & {$[84,85,86,87,88,89,90,91,27,93,94,95,52]$} & $22: 00-7: 00$ \\
& & &
\end{tabular}

It is assumed that the substation 1 has a main transformer overhaul, and the branch 27 and the branch 88 also have the need of overhaul. Node 51 has the need for power protection. According to the previous analysis, there are branch 27, 88 disconnected, branch 52 is dis-connected. Substation 1 has two main transformers, each with a capacity of 12.5 MVA. Substation 2 and Substation 3 are also two main transformers, each with 8 MVA. Set up a switchable capacitor at node 24/42/72, a total of 4 switchable groups, each group with a switching capacity of $50 \mathrm{kVar}$, reconfigure the distribution network, and optimize the grid reconstruction scheme. The capacitor switching plan is shown in Table 1.

Scheme 1 uses the network loss of the distribution network as the objective function. The system network loss value is $3189.370 \mathrm{kWh}$ and the load balance index value is 0.2734 . Scheme 2 uses the load balance of the distribution network as the objective function, and the system network loss value is $3286.157 \mathrm{kWh}$. The load is balanced. The index value is 0.2187 . In the actual operation of the distribution network, the line loss is the focus of people's attention, so the first program in the actual operation of the power grid, use more.

Based on the above analysis, we can see that the comprehensive optimization strategy of distribution network operating efficiency proposed in this paper can not only reduce the loss of distribution network, improve the economic efficiency of distribution network, but also make the load distribution of the power grid more balanced, for the distribution network operating efficiency. The promotion has a positive effect. 


\section{Conclusion}

This paper studies and establishes a comprehensive optimization model of distribution network operating efficiency. Firstly, the characteristics of the distributed power supply in the distribution network are analyzed. Based on this, the network reconstruction and DG active power output adjustment methods are used to comprehensively optimize the distribution network operating efficiency. Finally, the particle swarm algorithm is used to solve the complex distribution of 94 nodes. The results show that the proposed method is real and effective.

\section{Acknowledgements}

This work was financially supported by State Grid Corporation Science and Technology Project, Research on Operation Efficiency Monitoring and Analysis Technology of Distribution Network Assets Operation Efficiency Based on Large Data(WBS NO: 524608160055).

\section{References}

[1] Cui Fengliang, Zhou Jiachun. Remote Automatic Backup Power Supply Device[J]. Electric Power Automation Equipment, 2002, 22(9): 61-62.

[2] Yu Kun, Cao Yijia, Ni Yixin, et al. Review of Distributed Generation Technology and Its Grid Operation[J]. Journal of Hohai University (Natural Science Edition), 2009, 37(6): 741-748.

[3] Morozumi S. Micro-grid Demonstration Projects in Ja-pan[C]// Power Conversion Conference Nagoya, 2007. PCC '07. IEEE, 2007:635-642.

[4] Zhao Jingjing, Li Xin, Peng Yi, et al. Integrated optimization algorithm for power distribution network reconstruction and distributed power injection based on particle swarm opti-mization algorithm [J]. Power System Technology, 2009, 33(17): 162-166 .

[5] Zhuang Yuan, He Hai, Yang Xiaohui, et al. Distribution Network Reconfiguration with Distributed Power Optimiza-tion Scheduling[J]. Power System and Clean Energy, 2012, 28(11): 13-18.

[6] Wu Y K, Lee C Y, Liu L C, et al. Study of Reconfiguration for the Distribution System With Distributed Generators[J]. IEEE Transactions on Power Delivery, 2010, 25(3):1678-1685.

[7] Wang Wei, Huang Dawei. Integrated Optimization of Dis-tribution Network with Dispatchable Distributed Power Supply[J]. Transactions of China Electrotechnical Society, 2015, 30(12): 429-433.

[8] Khodr H M, Martinez-Crespo J, Matos M A, et al. Distribu-tion Systems Reconfiguration Based on OPF Using Benders Decomposition[J]. IEEE Transactions on Power Delivery, 2009, 24(4):2166-2176.

[9] Changzhi Power Supply Company Transformer Room: 220kV Changzhi West Station No. 1 and No. 2 main trans-former round stop maintenance work completed success-fully [EB/OL]. 2014-11-14.

[10] Liu Zhenya. China Power and Energy [M]. Beijing: China Electric Power Press, 2012: 102.

[11] Li Peng, Dou Pengchong, Li Yuwei, et al. Application of Microgrid Technology in Active Distribution Network [J]. Electric Power Automation Equipment, 2015, 35(4): 8-16.

[12] Ai Xin, Xu Jiajia. Research on Coordinated Operation of Microgrid and Distribution Network Based on Interactive Scheduling[J]. Power System Protection and Control, 2013, 41(1): 143-149.

[13] Zhou Suwen. Optimal distribution and reconstruction of distributed power distribution networks[D]. Nanjing: Hohai University, 2012. 